\title{
Hearing Preservation Surgery via Retrosigmoid Approach with Retrolabyrinthine Meatotomy in Small Vestibular Schwannoma
}

\author{
Diego Cazzador ${ }^{1}$ Daniele Borsetto ${ }^{1}$ Enrico Alexandre ${ }^{1}$ Chiara Pavone $^{1}$ Domenico d'Avella ${ }^{2}$ \\ Antonio Mazzoni ${ }^{1} \quad$ Elisabetta Zanoletti ${ }^{1}$
}

${ }^{1}$ Section of Otorhinolaryngology, Department of Neurosciences, University of Padua, Padua, Italy

${ }^{2}$ Section of Neurosurgery, Department of Neurosciences, University of Padua, Padua, Italy

\begin{abstract}
Address for correspondence Diego Cazzador, MD, Section of Otorhinolaryngology, Department of Neurosciences, University of Padua, Via Giustiniani 2, 35128, Padova, Italy (e-mail: gkmcaz@hotmail.it).
\end{abstract}

J Neurol Surg B 2019;80(suppl S3):S281-S283.

\begin{abstract}
Keywords

- acoustic neuroma

- hearing preservation surgery

- retrolabyrinthine meatotomy

- retrosigmoid approach

- vestibular schwannoma

Objectives Vestibular schwannomas (VS) extending into the internal auditory canal (IAC) are currently considered as an unfavorable condition for hearing preservation surgery (HPS) via retrosigmoid (RS) approach due to the poor direct visualization of the facial and cochlear nerves course through the IAC and the fundus during microsurgery. Design The operative steps are described in a surgical instructional video.

Setting The surgery took place at a tertiary-care center.

Participants Patient is a 45-year-old man who was incidentally diagnosed with an extrameatal VS extending for $9 \mathrm{~mm}$ into the left cerebellopontine angle (CPA). Hearing function at diagnoses was excellent, with pure tone average (PTA) $=15 \mathrm{~dB}$ and speech discrimination score (SDS) $=100 \%$ (class A according to the Tokyo classification) and minimal impairments on auditory brainstem response ( $A B R$ ). Given these preoperative features in small VS, hearing was the main function to look into in the treatment planning. Initial observation or HPS were proposed.

Results The patient underwent surgical excision for HPS via RS approach combined with retrolabyrinthine meatotomy (RLM). RLM enables the complete exposure of the IAC to the fundus, after drilling the bony surface of the posterior IAC wall, under guidance of the anatomical landmarks, namely, the endolymphatic duct, the blue lines of the posterior, and superior semicircular canals, and common crus. Nor intra-, neither postoperative complications occurred. Histologic examination confirmed the diagnosis of VS. A 3-month short-term follow-up revealed a class B hearing function with $\mathrm{PTA}=30 \mathrm{~dB}, \mathrm{SDS}=100 \%$, and normal facial nerve status.

Conclusions RLM via RS approach proved to be effective for HPS, enabling the full course of the facial and cochlear nerves through the IAC to be directly exposed. The link to the video can be found at: https://youtu.be/KC1S4pxpLCk.
\end{abstract}

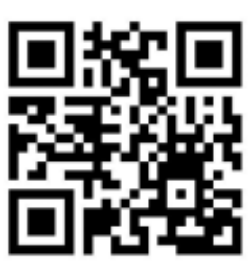

received

June 1, 2018 accepted after revision November 11, 2018 published online February 28, 2019

\section{Conflict of Interest}

None.

www.thieme.com/skullbasevideos

www.thieme.com/jnlsbvideos

DOI https://doi.org/

10.1055/s-0039-1677847. ISSN 2193-6331.
๑) 2019 Georg Thieme Verlag KG
Stuttgart · New York

License terms

(c) (1) $\ominus$ (\$) 


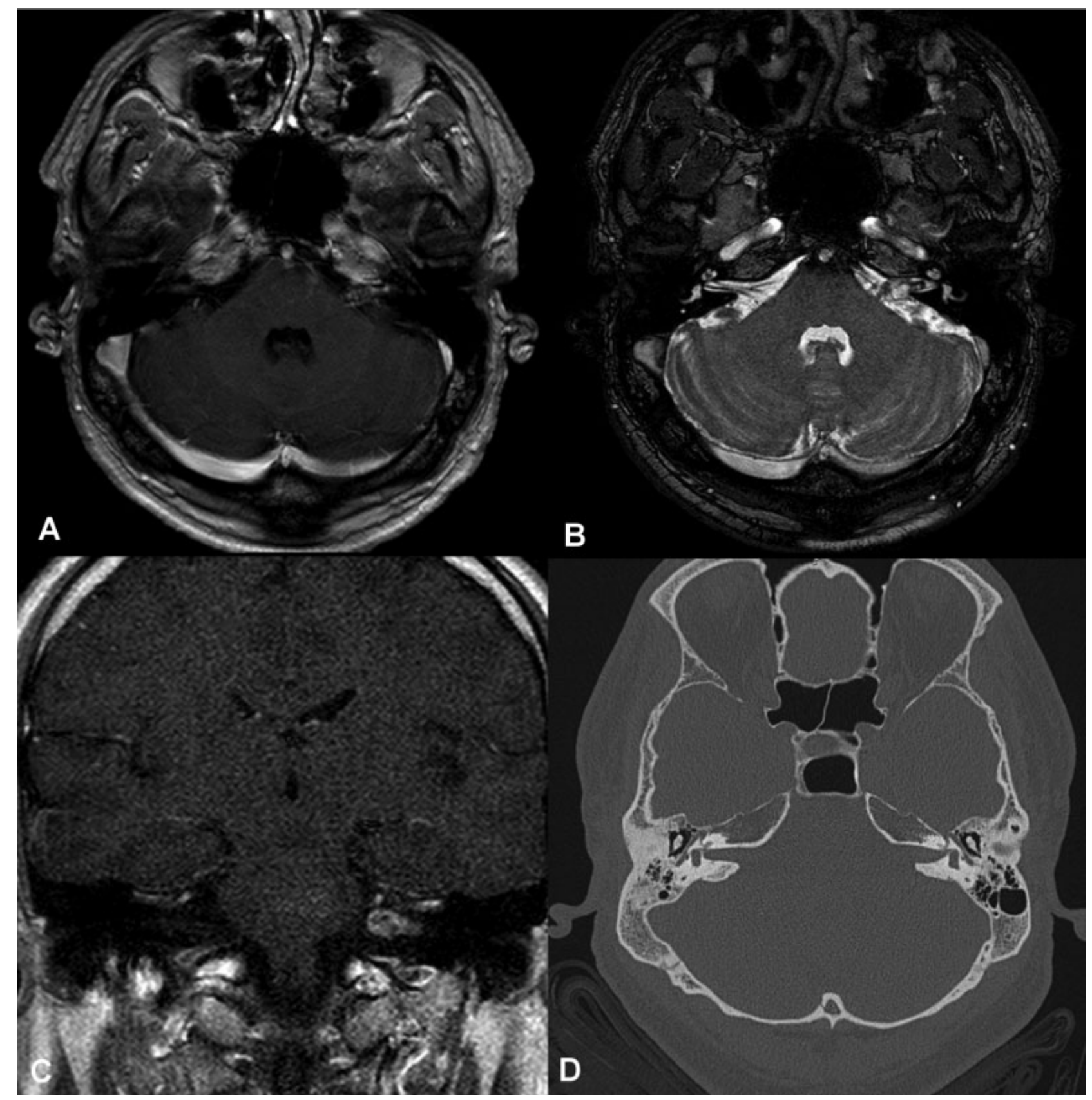

Fig. 1 (A) T1-weighted axial MRI scan with gadolinium, (B) axial 3D-CISS scan, and (C) coronal T1-weighted MRI scan with gadolinium show a contrast-enhancing lesion in the left internal auditory canal with a 9-mm-cystic extension into the cerebellopontine angle, suggestive for a vestibular schwannoma with extrameatal cystic component. On axial 3D-CISS scan (B), a cystic part of the extrameatal portion of the vestibular schwannoma is evident; the lateral part of the tumor is 1-2 mm apart to the fundus of the internal auditory canal. (D) Axial CT scan of the temporal bone revealed a slightly enlarged left internal auditory canal. 3D, three-dimensional; CISS, constructive interference in steady state; $\mathrm{CT}$, computed tomography; MRI, magnetic resonance imaging. 


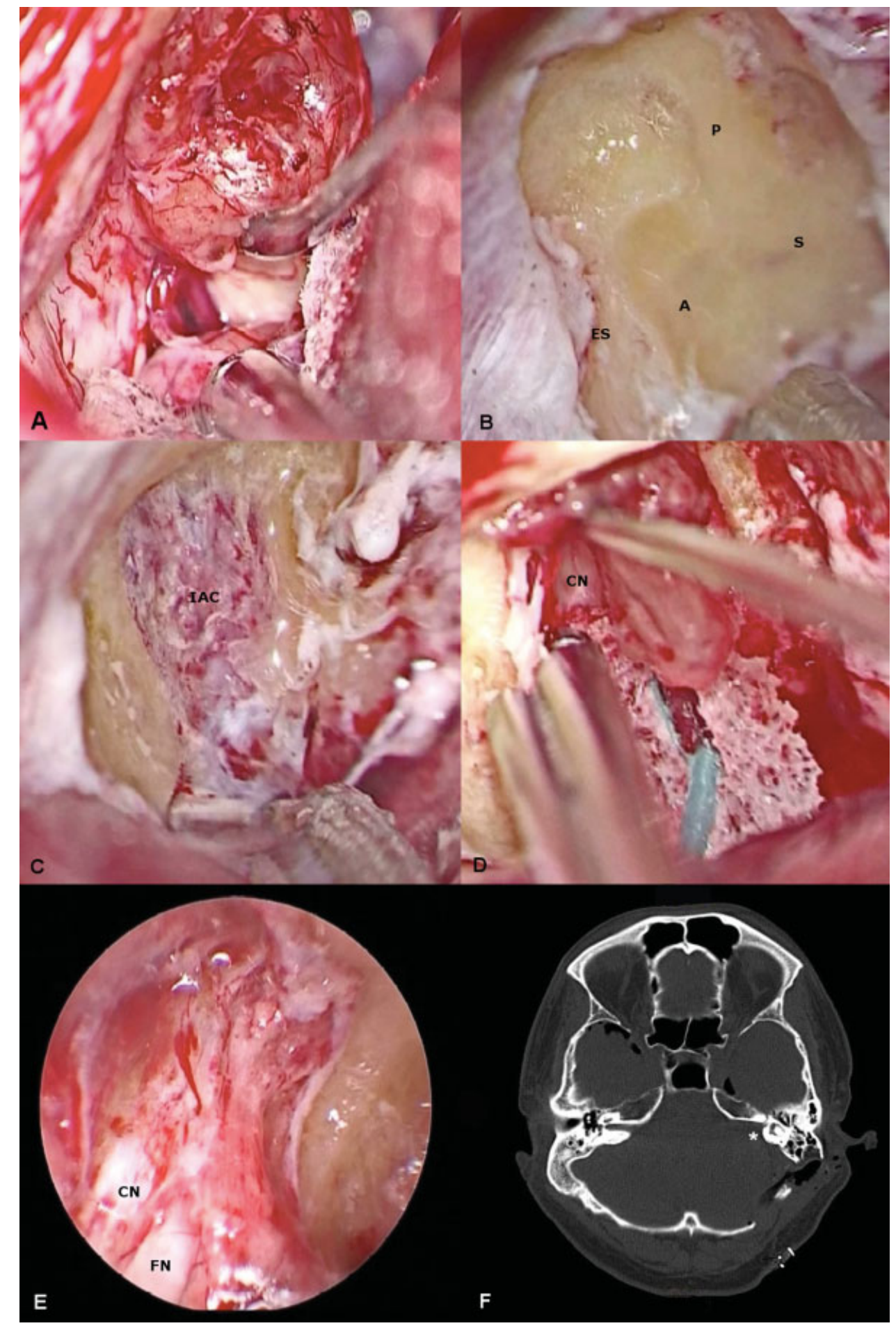

Fig. 2 Left side. Intraoperative microscopic view of the surgical field (A-D). The extrameatal component of the vestibular schwannoma is gently dissected from the VIII cranial nerve (A). Bony landmarks of the retrolabyrinthine meatotomy (B) are represented by the blue lines of the posterior semicircular canal $(P)$ and the superior semicircular canal (S), the endolymphatic aqueduct (A), and the endolymphatic sac (S). After drilling the posterior wall of the internal auditory canal (IAC), its posterior dural layer is exposed (C). Dissection of the intrameatal portion of the tumor from the cochlear nerve (CN), at the fundus of the IAC (D). Intraoperative endoscopic inspection of the fundus reveals the absence of residuals, as well as intact CN and facial nerve (FN). (E). Postoperative CT scan shows the absence of the posterior wall of the IAC on the left side ${ }^{*}$ ), as result of the retrolabyrinthine meatotomy performed via retrosigmoid approach (F). CT, computed tomography, IAC, internal auditory canal. 